\title{
Cuando la curiosidad científica se transforma en un videotutorial para aprender enseñando: conocimiento del contenido, elaboración de las explicaciones y complejidad de las preguntas
}

\author{
Jesús Ribosa ${ }^{1}$ \\ David Duran ${ }^{1}$ \\ ${ }^{1}$ Universitat Autònoma de Barcelona (UAB), España
}

Resumen. La creación de materiales didácticos por parte de los estudiantes es una forma de aprender enseñando, que ofrece una situación comunicativa al establecer una audiencia y puede contribuir a activar mecanismos de elaboración del conocimiento. Ante la escasa, pero prometedora, práctica de este tipo de propuestas educativas en educación primaria, se ha diseñado el Proyecto Bikos, en el que parejas de estudiantes elaboran cooperativamente videotutoriales a partir de su curiosidad científica. A partir de esta innovación, se plantean tres objetivos de investigación: 1) Examinar si se producen mejoras en el conocimiento específico sobre la pregunta que los estudiantes responden en cada videotutorial; 2) Estimar el grado de elaboración del conocimiento en las explicaciones que los estudiantes ofrecen en sus videotutoriales; y, 3) Analizar el grado de complejidad de las preguntas de comprensión para la audiencia que los estudiantes añaden a lo largo de sus videotutoriales. Los resultados de un pretest-posttest muestran mejoras significativas en el conocimiento específico. El análisis exploratorio de las explicaciones sugiere que cerca de dos tercios de la información ha sido suficientemente elaborada por los estudiantes. El análisis de las preguntas de comprensión señala que su gran mayoría tiene un bajo nivel de complejidad.

Palabras clave: aprender enseñando; aprendizaje cooperativo; enseñanza de las ciencias; material didáctico; vídeo educativo.

Quando a curiosidade científica se transforma em um vídeo tutorial para aprender ensinando: conhecimento do conteúdo, elaboração das explicações e complexidade das perguntas

Resumo. A criação de materiais didáticos pelos estudantes é uma forma de aprender ensinando, que oferece uma situação comunicativa ao estabelecer um público e pode contribuir para ativar mecanismos para a elaboração do conhecimento. Diante da escassa, mas promissora, prática deste tipo de propostas educacionais no ensino primário (anos iniciais do ensino fundamental), foi elaborado o Projeto Bikos, no qual pares de alunos criam, cooperativamente, vídeos tutoriais baseados na curiosidade científica. A partir desta inovação, propõem-se três objetivos de pesquisa: 1) examinar se há melhorias no conhecimento específico sobre a pergunta respondida pelos estudantes em cada vídeo tutorial; 2) estimar o grau de elaboração do conhecimento nas explicações oferecidas pelos alunos nos vídeos tutoriais; e 3) analisar o nível de complexidade das perguntas de compreensão feitas pelos estudantes para o público ao longo dos seus vídeos tutoriais. Os resultados de um pré-teste e pós-teste mostram melhoras significativas em conhecimentos específicos. A análise exploratória das explicações indica que cerca de dois terços das informações foram suficientemente elaboradas pelos estudantes. A análise das perguntas de compreensão aponta que a grande maioria delas tem um baixo nível de complexidade

When scientific curiosity turns into a video tutorial for learning by teaching: content knowledge, elaboration of the explanations, and complexity of the questions

Abstract. The creation of teaching materials by students is a way of learning by teaching, which offers a communicative situation by setting an audience and can contribute to activating mechanisms for knowledge elaboration. Given the scarce, but promising, practice of this kind of educational proposals in primary education, Bikos Project has been designed, in which pairs of students cooperatively create video tutorials based on their scientific curiosity. Three research aims are addressed: 1) To examine if there is any improvement in the specific knowledge about the question that the students answer in each video tutorial; 2) To estimate the degree of knowledge elaboration in the explanations that students offer in their video tutorials; and 3) To analyze the degree of complexity of the comprehension questions for the audience that students add throughout their video tutorials. The results of a pretest-posttest show significant improvements in specific knowledge. The exploratory analysis of the explanations suggests that about two thirds of the information has been sufficiently elaborated by students. The analysis of the comprehension questions indicates that the vast majority show a low level of complexity.

Keywords: cooperative learning; educational video; learning by teaching; science education; teaching materials. 


\section{Introducción}

Cada vez resulta más evidente la necesidad de que las prácticas de enseñanza y aprendizaje de las ciencias permitan que los estudiantes hablen de ciencia, es decir, formulen verbalmente explicaciones para comunicar y argumentar ideas científicas (Bennett et al., 2010; Colley y Windschitl, 2016; S. W. Lin et al., 2016; Ødegaard et al., 2016). El relevante papel del lenguaje en la construcción del conocimiento ha sido ampliamente argumentado en los postulados socioconstructivistas (Mercer, 2000; Vygotsky, 1978). En el área de ciencias, en todas las etapas educativas, numerosas propuestas plantean que los estudiantes verbalicen ideas científicas, ya sea al exponer delante de la clase o de otra audiencia (p. ej., Perales y Vílchez, 2015; Rosa y Martínez-Aznar, 2019), o al interactuar con sus compañeros cuando resuelven una tarea cooperativamente (p. ej., Durán-García y Durán-Aponte, 2013; Pavón y MartínezAznar, 2014; Reigosa y Jiménez, 2011). El aprendizaje cooperativo es una de las estrategias didácticas más utilizadas e investigadas para el cambio conceptual (J. W. Lin et al., 2016). Uno de los temas centrales de investigación sobre el aprendizaje de las ciencias tiene que ver con las concepciones cotidianas de los estudiantes, consideradas habitualmente como representaciones que deben ser sustituidas por explicaciones científicas correctas (Furberg y Arnseth, 2009). El aprendizaje que tiene lugar cuando se revisan estas concepciones alternativas previas se conoce como cambio conceptual (Mason y Zaccoletti, 2020), constructo que sigue siendo considerablemente investigado (T. J. Lin et al., 2019). El aprendizaje cooperativo se considera una estrategia privilegiada por su potencial para que los estudiantes, al verbalizar sus ideas científicas, hagan emerger perspectivas divergentes en la interacción y generen así conflictos sociocognitivos (p. ej., Can y Boz, 2016; De la Hera et al., 2019; Eymur y Geban, 2017).

El desarrollo y la difusión de las Tecnologías Digitales (TD) ofrecen nuevas posibilidades para comprender y expresar información en múltiples lenguajes y formatos (Coll, 2013). Tienen a su vez un gran impacto en la cantidad de información accesible, así como en el número y la diversidad de contextos donde participar (Coll, 2013; Collins y Halverson, 2010). El uso de las TD en el aula puede ser beneficioso si las oportunidades que ofrecen se aprovechan para potenciar los principios del aprendizaje efectivo (Yeung et al., 2021). Actualmente, uno de los principales retos de la educación es cómo educar en el marco de una cultura digital (Coll y Rodríguez, 2008; Ilomäki et al., 2016).

Uno de los componentes principales de la alfabetización digital es el proceso de búsqueda de información, cuya regulación consciente es un elemento clave (Coiro, 2011; Coiro y Dobler, 2007; Goldman et al., 2012; Monereo y Fuentes, 2008). Para que los estudiantes actúen estratégicamente, es necesario planear formas de apoyo a lo largo del proceso, especialmente en educación primaria y secundaria (Coiro y Dobler, 2007; Knight y Mercer, 2015; Macedo-Rouet et al., 2013; Zhang y Quintana, 2012). El desarrollo de estrategias de búsqueda de información juega un papel importante en la alfabetización científica. Una de las competencias científicas evaluadas en PISA es la de explicar fenómenos científicamente, es decir, "reconocer, ofrecer y evaluar explicaciones para un abanico de fenómenos naturales y tecnológicos" (Organización para la Cooperación y el Desarrollo Económicos [OCDE], 2019, p. 100). A 
diario las personas buscan intencionadamente o se encuentran accidentalmente con afirmaciones científicas procedentes de distintas fuentes (Sinatra y Lombardi, 2020). Las investigaciones muestran cuán difícil es evaluar esas afirmaciones al leer en internet (Sinatra y Hofer, 2016). Leer en línea es más complejo porque no solamente implica la construcción interna de significado, sino también un proceso autodirigido de construcción del texto a través de la selección de webs y enlaces (Coiro y Dobler, 2007; Pozo, 2014).

El uso de las TD en el aula exige a los docentes llevar a cabo un razonamiento pedagógico más complejo para planificar, implementar y evaluar sus prácticas educativas (Webb y Cox, 2004). Además del acceso a dispositivos, cabe considerar también el conocimiento tecnológico, pedagógico y del contenido que tiene el docente, así como las creencias, actitudes y emociones que pueden estar detrás de la decisión de utilizar o no las TD en clase (Howard, 2013; Petko, 2012; Voogt et al., 2012). En el aula de ciencias, la creación de vídeos por parte de los estudiantes es una práctica cada vez más habitual (Farrokhnia et al., 2020; Gallardo-Williams et al., 2020; Reyna y Meier, 2018; Snelson, 2018). Estos vídeos pueden elaborarse para ofrecer una explicación a una audiencia real, potencial o ficticia -es decir, pueden tomar la forma de materiales didácticos-. La creación de materiales didácticos para que otros potenciales aprendices también puedan aprender el contenido es una forma de aprender enseñando -o aprenseñar- (Duran, 2014). Si este material se elabora para ponerlo a disposición del potencial aprendiz, y no como apoyo a una explicación, nos situaríamos en el marco del primer nivel de aprenseñar: aprender con la expectativa de que alguien utilizará ese material para aprender (Duran, 2014). Esta expectativa generada en la situación de aprender para enseñar -aunque finalmente no se enseñe- se conoce como expectancy (Bargh y Schul, 1980; Benware y Deci, 1984). Las investigaciones sobre este constructo indican que, al menos bajo determinadas condiciones, la expectativa de enseñar puede generar procesos de revisión del contenido, organización para presentarlo e identificación de su estructura básica (Duran, 2014, 2017; Kobayashi, 2019) y promover una mayor motivación (Fiorella y Mayer, 2014; Hoogerheide et al., 2019). La creación de materiales didácticos también podría generar procesos vinculados a los otros niveles de aprenseñar: aprender y exponer, aprender y explicar, y aprender y explicar mediante preguntas (Duran, 2014, 2017).

En educación obligatoria, el número de artículos de investigación que analizan la creación de vídeos educativos por parte de los estudiantes es muy reducido (Ribosa y Duran, 2021). En educación primaria, Penttilä et al. (2016) investigan la creación de breves historias digitales en las que los estudiantes explican experimentos científicos en el área de química. Los resultados sugieren que los estudiantes que crean historias digitales muestran un mayor nivel de abstracción sobre los contenidos de aprendizaje. En otra investigación, Hoogerheide et al. (2019) piden a los estudiantes que generen como deberes un vídeo educativo a partir de un texto sobre biología. En comparación con volver a estudiar el material o hacer un resumen, los estudiantes percibieron la tarea como más divertida. En cuanto al conocimiento conceptual, no se encontraron diferencias significativas entre crear el vídeo o hacer un resumen. Aun así, de estos dos tratamientos, solo el grupo que creó el vídeo obtuvo diferencias significativas con respecto al grupo de control que estudió el material. En matemáticas existen algunos 
estudios en educación primaria que reportan beneficios de aprendizaje a través de la creación de vídeos, al analizar los productos generados por los estudiantes (Soto, 2015) y también el proceso de representación mental del problema (Muis et al., 2016). También en educación secundaria encontramos algunos estudios de creación de vídeos en áreas como ciencias de la tierra (Mills, Tomas y Lewthwaite, 2020), biología (Jablonski et al., 2015) o física (Downie et al., 2017). Aunque algunos de estos estudios se sitúan en la perspectiva de aprender enseñando (Hoogerheide et al., 2019; Muis et al., 2016), la mayoría de las investigaciones sobre la creación de materiales didácticos por parte de los estudiantes no atribuyen explícitamente los beneficios a la actividad de aprender enseñando (Ribosa y Duran, 2021). Es necesario diseñar intervenciones que partan de esta perspectiva para maximizar sus beneficios y anticipar posibles dificultades (Duran, 2014, 2017).

Ante la escasa, pero prometedora, práctica de propuestas educativas basadas en la creación de materiales didácticos por parte de los estudiantes en educación primaria, se ha diseñado el Proyecto Bikos, objeto y contexto de estudio del presente artículo. En un formato para desarrollar la alfabetización digital y el aprendizaje autodirigido, la propuesta didáctica plantea que parejas de estudiantes formulen preguntas científicas, a partir de sus propios intereses y curiosidad por conocer el mundo, y creen videotutoriales como mecanismo para su propio aprendizaje. Ante el carácter dinámico del conocimiento, es necesario que la escuela no se limite a transmitir respuestas, sino que enseñe a hacerse preguntas y a desarrollar estrategias para encontrarles respuesta (Fernández-Enguita, 2017). Como señalan Freire y Faundez (2010), el inicio del conocimiento es la pregunta, y solo a partir de esta se debe iniciar la búsqueda de respuestas. Además, la posibilidad de elección sobre lo que se aprende, para conectarlo con los intereses y experiencias del aprendiz, es una de las implicaciones de la personalización del aprendizaje (Coll, 2018).

En el marco de este proyecto, en el presente artículo se plantean los siguientes objetivos:

1. Examinar si se producen mejoras en el conocimiento específico sobre la pregunta que los estudiantes responden en cada videotutorial.

2. Estimar el grado de elaboración del conocimiento en las explicaciones que los estudiantes ofrecen en sus videotutoriales.

3. Analizar el grado de complejidad de las preguntas de comprensión para la audiencia que los estudiantes añaden a lo largo de sus videotutoriales.

\section{Métodos y materiales}

Para dar respuesta a estos tres objetivos, se adoptó un enfoque metodológico mixto. Se llevó a cabo un diseño pretest-posttest de un solo grupo, en el que se implementó el proyecto de intervención -descrito en el siguiente subapartado-. Con el análisis del pretest-posttest se pretende examinar si se producen mejoras en el conocimiento específico sobre las preguntas -primer objetivo-. Este diseño preexperimental se combina con un análisis exploratorio de los videotutoriales elaborados, para explicar las posibles diferencias entre el pretest y el posttest. Este análisis exploratorio pone el foco en el grado de elaboración de las explicaciones -segundo objetivo- y el grado de complejidad de las preguntas de comprensión -tercer objetivo-. 


\subsection{Descripción del proyecto}

Desde el área de conocimiento del medio, el Proyecto Bikos pretende contribuir al desarrollo de una serie de competencias del marco curricular catalán (Decreto 119/2015), especialmente las vinculadas a aprender a aprender, al ámbito digital y al tratamiento de la información. Se ha diseñado para que pueda implementarse en ciclo superior de educación primaria y primer ciclo de educación secundaria obligatoria. La propuesta didáctica plantea que parejas de estudiantes elaboren un videotutorial para responder una pregunta sobre el mundo, que ellos mismos formulan. En esta propuesta, el concepto de videotutorial intenta alejarse del modelo transmisivo procedimental que normalmente encontramos en las redes. Entendemos el videotutorial como un vídeo de máximo tres minutos en el que el contenido se presenta acompañando a la audiencia en su proceso de comprensión, mediante la formulación de preguntas de conocimientos previos al inicio, preguntas de comprensión durante el videotutorial y una pregunta de comprensión global al final. Se trata pues de crear un material que permite a potenciales aprendices aprender lo que estos estudiantes han aprendido previamente; por lo tanto, de aprender enseñado en expectancy (Duran, 2017; Fiorella y Mayer, 2014).

En una organización del aula basada en el aprendizaje cooperativo (Topping, et al. 2017), se propone que los estudiantes trabajen en parejas, con dos roles complementarios: responsable de contenido y responsable tecnológico. Los estudiantes disponen de dos materiales que les ayudan a estructurar su actividad conjunta: una guía de roles y una hoja de actividad, que contemplan las siguientes fases:

0. Definición de la pregunta. Cada pareja define la pregunta para su videotutorial. Aunque es el responsable de contenido quien lleva la iniciativa en la formulación de la pregunta, es necesario un proceso de negociación entre ambos miembros para que la pregunta los motive e implique en la creación del videotutorial.

1. Conocimientos previos. Cada miembro de la pareja elabora una respuesta conjetural a la pregunta planteada, es decir, propone una explicación hipotética del fenómeno -basándose en sus conocimientos previos, sin consultar fuentes de información-. Seguidamente, comparten sus respuestas y definen dudas.

2. Búsqueda de información. En un proceso recursivo, los estudiantes definen los conceptos clave de la búsqueda; buscan, seleccionan y contrastan diferentes fuentes de información; resuelven dudas iniciales y se plantean nuevas cuestiones. Este proceso concluye con la elaboración de una respuesta compartida a la pregunta planteada.

3. Elaboración del guion. Los estudiantes elaboran el guion del vídeo, en el que organizan y transforman la información con el objetivo de presentarla a una audiencia potencial real a través de un videotutorial. Esto incluye la redacción de la narración del videotutorial, y también la planificación de los recursos visuales y de las preguntas de conocimientos previos al inicio, las preguntas de comprensión durante el videotutorial y la pregunta de comprensión global al final. 
4. Producción del vídeo. Los estudiantes crean el videotutorial a partir del guion. Esta fase consta de dos periodos: generación de recursos y edición técnica; y edición pedagógica, para introducir las preguntas interactivas. En la implementación descrita en el presente artículo, se utilizó PowerPoint para la edición técnica del vídeo y EDpuzzle para la edición pedagógica, pero también podrían utilizarse otras herramientas tecnológicas en función del contexto de implementación.

Se propone llevar a cabo dos rondas de creación de videotutoriales con el grupo clase. Entre una y otra se intercambian los roles de la pareja, de forma que ambos miembros tengan la oportunidad de llevar la iniciativa al formular la pregunta en la que quieren indagar. Cada ronda de creación de videotutoriales tiene una duración aproximada de 12 horas de clase. Los videotutoriales resultantes serán organizados en un espacio virtual a disposición de otros estudiantes y centros educativos.

\subsection{Contexto y participantes}

Después de llevar a cabo una prueba piloto en el curso 2017-2018, en el curso 2018-2019 el proyecto fue implementado en dos centros públicos de educación primaria de Catalunya. Se requería que los centros dispusieran de los recursos digitales suficientes -un ordenador o tableta por pareja, con conexión a internet- para implementar el proyecto. Sin embargo, se quería que no llevaran a cabo frecuentemente prácticas en las que los estudiantes usaran tecnologías digitales. Participaron en el proyecto un total de 44 estudiantes de sexto de primaria, es decir, 22 parejas, que crearon dos videotutoriales cada una. Se llevó a cabo una formación inicial de cuatro docentes, así como reuniones para ajustar la implementación del proyecto al contexto de cada centro. Luego, los estudiantes recibieron una formación inicial por parte de los docentes para familiarizarse con los objetivos del proyecto, el tipo de producto, el proceso de creación y los materiales de apoyo. Durante la implementación del proyecto, docentes e investigadores colaboraron para llevarlo a cabo en las aulas y evaluarlo en dos rondas de creación de videotutoriales. Uno de los investigadores asistió como observador participante a todas las sesiones del proyecto de intervención en ambas escuelas, para ayudar a los docentes a organizar el aula y ofrecer apoyo especialmente en las cuestiones tecnológicas.

Los estudiantes formularon en sus videotutoriales preguntas muy diversas, sobre el universo (p. ej., ¿qué es el Big Bang?, ¿cómo se formó la Vía Láctea?), sobre fenómenos del entorno natural (p. ej., ¿cómo se forma la nieve?, ¿por qué los atardeceres tienen diferentes colores?), sobre animales y plantas (p. ej., ¿por qué los pandas están en peligro de extinción?, ¿por qué motivos la luz puede mover las plantas?), sobre fenómenos del entorno social (p. ej., ¿por qué hay tantos idiomas en el mundo?, ¿qué es la mitología nórdica?), sobre los seres humanos (p. ej., ¿cómo funciona el cerebro?, ¿por qué los humanos tenemos sentimientos?) y sobre tecnología (p. ej., ¿cómo calcula la calculadora?, ¿qué inteligencias artificiales son más inteligentes que la humana?). Como se puede ver, muchas de ellas abordan cuestiones interdisciplinares. ${ }^{1}$

\footnotetext{
${ }^{1}$ Pueden consultarse dos ejemplos de videotutoriales elaborados por estudiantes: Per què el cel és blau? (en catalán, ¿Por qué el cielo es azul?, disponible en https://edpuzzle.com/media/5c8bbf4adedf7c409e552be8), ¿La luz interviene en cómo vemos los colores? (en castellano, disponible en https://edpuzzle.com/media/5c5027287fa15e411ebffbf7).
} 


\subsection{Recogida y análisis de datos}

Para el primer objetivo -examinar si se producen mejoras en el conocimiento específico sobre las preguntas-, se llevó a cabo un pretest-posttest, en el que los estudiantes respondían a su pregunta sin poder acceder a fuentes de información antes y después de la creación de cada videotutorial. Las respuestas se evaluaron con una rúbrica de seis ítems, elaborada a partir del modelo de Zhu et al. (2009): completitud de la información, comprensibilidad, pertinencia, veracidad, grado de detalle y apariencia de experto. La rúbrica fue validada por tres jueces. En los distintos ítems se obtuvieron valores entre 0,61 y 0,86 en el coeficiente kappa de Cohen ponderado. Después de evaluar las respuestas de los estudiantes, se compararon las puntuaciones del pretest y del posttest de todos los videotutoriales, mediante la prueba de los rangos con signo de Wilcoxon. Esta prueba también se llevó a cabo para comparar la extensión de las respuestas del pretest y las del posttest.

Para el segundo objetivo -estimar el grado de elaboración del conocimiento en las explicaciones-, se transcribieron las explicaciones que los estudiantes desarrollaron en sus videotutoriales, así como cualquier texto sobreimpreso que añadieran en las imágenes. Para la estimación del grado de elaboración de las explicaciones, se utilizó la herramienta de detección de plagio Quetext, por su avanzada tecnología que le permite llevar a cabo un análisis contextual y detectar cambios superficiales (https:// www.quetext.com/). Como esta herramienta no detecta plagios en los textos y fuentes en catalán, la lengua utilizada por los estudiantes -junto al castellano-, se tomaron dos medidas adicionales: a) se tradujeron al castellano con el traductor de Google -y posterior revisión- las transcripciones de aquellos videotutoriales que se encontraban en catalán para poder analizarlas con Quetext; y b) se identificaron los videotutoriales en los que los estudiantes reportaron haber utilizado alguna fuente de información en catalán. En estos casos en los que se utilizaron fuentes de información en catalán, las transcripciones originales fueron analizadas con otra herramienta de detección de plagio: la de SmallSEOTools (https://smallseotools.com/es/plagiarism-checker/). Si bien esta no utiliza una tecnología tan avanzada como Quetext, sí que funciona correctamente con los textos y fuentes en catalán. En caso de que el porcentaje de plagio de una trascripción detectado con esta herramienta fuera superior al detectado con Quetext, este porcentaje pasaba a ser el que tenía validez para análisis posteriores con el conjunto de las transcripciones. En estos análisis se reportaron los estadísticos descriptivos siguientes: mínimo, máximo, media, desviación estándar y percentiles.

Para el tercer objetivo -analizar el grado de complejidad de las preguntas de comprensión para la audiencia-, se transcribieron los enunciados de las preguntas de comprensión que los estudiantes habían añadido a lo largo de sus videotutoriales. Se excluyeron del análisis las otras dos preguntas: la pregunta de conocimientos previos al inicio y la pregunta de comprensión global al final. También se excluyeron del análisis las opciones de respuesta -correctas y distractores- y la retroalimentación posterior que algunos estudiantes habían añadido para la audiencia. A partir de la taxonomía revisada de Bloom (Anderson y Bloom, 2001), el enunciado de cada pregunta de comprensión se codificó en una de las siguientes categorías, considerando también la información que los estudiantes aportaban en la explicación del videotutorial: recordar, comprender, aplicar, analizar, evaluar, crear (Tabla 1). 
Tabla 1. Definición de las categorías de la taxonomía revisada de Bloom

Definición

Crear Juntar elementos para formar un todo coherente o funcional; reorganizar elementos en un nuevo patrón o estructura.

Evaluar Emitir juicios a partir de criterios o normas.

Analizar Descomponer el material en sus partes constituyentes y determinar cómo las partes se relacionan entre ellas y con una estructura o propósito generales.

Aplicar Llevar a cabo o utilizar un procedimiento en una situación dada.

Comprender Construir significado a partir de mensajes instructivos, incluyendo comunicación oral, escrita y gráfica.

Recordar Recuperar conocimiento relevante de la memoria a largo plazo.

Fuente: extraída de Anderson y Bloom (2001).

Se reportaron frecuencias, porcentajes y ejemplos de las categorías identificadas. Aunque las orientaciones del proyecto dictaban que estas preguntas de comprensión fueran de opción múltiple, se revisaron todas para identificar si los estudiantes habían utilizado también otros formatos.

\section{Resultados}

\subsection{Conocimiento específico sobre la pregunta}

Los resultados muestran que los estudiantes mejoran significativamente su nivel de conocimiento específico sobre las preguntas que se plantean en sus videotutoriales (Tabla 2). Al no disponer de grupos de comparación, no podemos atribuir con seguridad estas mejoras a la intervención. Sin embargo, al tratarse de un conocimiento tan específico, que no se trabaja en otras situaciones de instrucción, asumimos que la participación en el proyecto puede ser la principal responsable de las mejoras.

Tabla 2. Análisis del pretest-posttest del conocimiento específico sobre las preguntas

\begin{tabular}{lccccc}
\cline { 2 - 6 } & $\begin{array}{c}\text { Media } \\
\text { pretest }\end{array}$ & $\begin{array}{c}\text { Media } \\
\text { posttest }\end{array}$ & $\begin{array}{c}\text { Diferencia } \\
\text { post-pre }\end{array}$ & $\begin{array}{c}\text { Desviación } \\
\text { estándar }\end{array}$ & $\begin{array}{c}\text { Sig. } \\
\text { (bilateral) }\end{array}$ \\
Completitud de la información & 0,64 & 1,48 & 0,84 & 0,741 & 0,000 \\
Comprensibilidad & 2,63 & 2,42 & $-0,21$ & 0,761 & 0,027 \\
Pertinencia & 2,13 & 2,00 & $-0,13$ & 1,429 & 0,424 \\
Veracidad & 0,94 & 1,59 & 0,65 & 0,983 & 0,000 \\
Grado de detalle & 0,13 & 1,08 & 0,95 & 0,870 & 0,000 \\
Apariencia de experto & 0,32 & 1,50 & 1,18 & 0,687 & 0,000 \\
Total & 6,77 & 10,07 & 3,30 & 3,025 & 0,000 \\
\hline
\end{tabular}

Fuente: elaboración propia.

De los seis ítems analizados, la completitud de la información, la veracidad, el grado de detalle y la apariencia de experto sí muestran mejoras significativas. No obstante, la pertinencia no muestra cambios significativos, y la comprensibilidad disminuye significativamente (Tabla 2). En cuanto a la pertinencia, cabe señalar que en el proceso de búsqueda los estudiantes encuentran grandes cantidades de infor- 
mación que no siempre se ajusta a su propósito. Si no regulan adecuadamente la selección de información, pueden incluir en sus respuestas no solo información clave para el propósito de la pregunta, sino también información anecdótica, que se desvía del foco principal. En referencia a la disminución significativa de la comprensibilidad, probablemente se deba a que las respuestas del posttest son considerablemente más extensas y sofisticadas que las del pretest. La media de palabras del pretest $(20,09)$ se sitúa muy por debajo de la media de palabras del posttest $(61,89)$. La prueba de significación estadística muestra que la extensión de las respuestas aumenta significativamente entre el pretest y el posttest $(p=0,000)$. Por ello, resulta más probable encontrar alguna idea cuya redacción no resulte clara, hecho que disminuye la puntuación de comprensibilidad.

\subsection{Grado de elaboración de las explicaciones}

Los resultados del análisis de las explicaciones muestran que, de media, un $37,48 \%$ (SD=24,72) de la información que aportan los estudiantes en las explicaciones podría considerarse plagio -es decir, no se encuentra suficientemente elaborada respecto a la fuente original- (Tabla 3).

Tabla 3. Estadísticos descriptivos de los porcentajes de plagio detectados en las explicaciones

\begin{tabular}{ccccccc}
\hline Mínimo & Máximo & Media & Desviación estándar & \multicolumn{3}{c}{ Percentiles } \\
& & & & 25 & 50 & 75 \\
0 & 82 & 37,48 & 24,72 & 16,5 & 38,5 & 57,5 \\
\hline
\end{tabular}

Fuente: elaboración propia.

El porcentaje de plagio podría ser mayor, ya que cabe tener en cuenta dos limitaciones. En primer lugar, la utilización de otros tipos de fuentes de información que no pueden ser detectadas tan fácilmente por las herramientas de detección de plagio -en 12 de los 44 videotutoriales los estudiantes reportan haber utilizado vídeos como fuentes de información-. En segundo lugar, la imposibilidad de la herramienta Quetext para contrastar la respuesta con fuentes de información en catalán -en 18 de los 44 videotutoriales los estudiantes reportan haber utilizado fuentes de información en catalán-. Solo en uno de estos dieciocho videotutoriales el uso del detector de plagio de SmallSEOtools identificó un porcentaje superior al de la herramienta Quetext. A pesar de estas limitaciones, cabe destacar que este análisis exploratorio sugiere que una media del $62,52 \%$ de la información ha sido suficientemente elaborada por los estudiantes a partir de la búsqueda de información.

\subsection{Grado de complejidad de las preguntas de comprensión para la audiencia}

En los 44 videotutoriales aparecen un total de 145 preguntas de comprensión. Se recomendaba a los estudiantes añadir una pregunta por cada apartado de su explicación, y como orientación se proponía organizar la explicación en tres o cuatro bloques. De las 145 preguntas, casi un 90\% pertenecen al nivel inferior de la taxonomía revisada de Bloom (Tabla 4). 
Tabla 4. Frecuencias, porcentajes y ejemplos de los tipos de preguntas de comprensión

\begin{tabular}{lccl}
\hline & $f$ & $\%$ & \\
Crear & 1 & 0,69 & ¿Qué podemos hacer para salvarlos [a los pandas]? \\
Evaluar & 0 & 0 & - \\
Analizar & 0 & 0 & - \\
Aplicar & 0 & 0 & - \\
Comprender & 13 & 8,97 & ¿Qué tienen las fosas marinas en común con las placas \\
& & & tectónicas? \\
Recordar & 129 & 88,97 & ¿Cuántos movimientos tiene la Tierra? \\
No codificable & 2 & 1,38 & ¿Ahora ya sabéis qué es el Big Bang? \\
\hline
\end{tabular}

Fuente: elaboración propia.

Las dos preguntas no codificables forman parte del mismo videotutorial, y adoptan un enfoque metacognitivo, al pedir a la audiencia que piense si ha entendido o no lo que se ha explicado (Tabla 4).

En cuanto al formato de las preguntas, aunque como orientación se recomendó que las preguntas de comprensión fueran de opción múltiple, 6 de las 145 preguntas de comprensión tienen un formato de respuesta abierta (p. ej., ¿ Te acuerdas del nombre de alguna mariposa de las que te hemos enumerado?).

\section{Discusión y conclusiones}

Parece que la participación de los estudiantes en el proyecto los lleva a mejorar significativamente su nivel de conocimiento específico sobre las preguntas que se plantean en los videotutoriales. Resulta necesario probar estas mejoras para estar convencidos de que se trata de un tiempo bien invertido, aún más al considerar la elevada complejidad de las preguntas que suelen plantearse los estudiantes. En el futuro, deberán compararse los resultados de los participantes en el proyecto con los de grupos de comparación. Al centrarnos en los seis ítems analizados en las respuestas iniciales y finales, hemos visto que se producen mejoras significativas en la completitud de la información, la veracidad, el grado de detalle y la apariencia de experto. La ausencia de cambios en la pertinencia de las respuestas probablemente se deba a la naturaleza abierta de los procesos de lectura en la red (Coiro y Dobler, 2007; Pozo, 2014). La disminución de la comprensibilidad puede tener su origen en la mayor extensión de las respuestas del posttest. En línea con estudios previos sobre intervenciones que plantean la creación de vídeos educativos, parece que los estudiantes mejoran su conocimiento al adoptar el rol de enseñante y tener que generar en los videotutoriales una explicación para otras personas, hecho que activa procesos metacognitivos (Hoogerheide et al., 2019; Penttilä et al., 2016; Soto, 2015).

En referencia al grado de elaboración del conocimiento en las explicaciones que los estudiantes ofrecen en sus videotutoriales, las estimaciones muestran que, de media, más del $60 \%$ de la información se encuentra suficientemente elaborada. En palabras de Roscoe y Chi (2007), podríamos considerar que en casi dos tercios de la información los estudiantes adoptan un enfoque de construir el conocimiento, es decir, 
lo ajustan para explicarlo a su audiencia potencial real a través del videotutorial; y en más de un tercio de la información adoptan un enfoque de decir el conocimiento, al transmitirlo a su audiencia con un bajo grado de elaboración. Cabe destacar aquí las limitaciones del uso de herramientas de detección de plagio, que en este estudio se han utilizado de forma exploratoria al contar con explicaciones de temas muy diversos que parten de las preguntas y de las fuentes que buscan los estudiantes. En el futuro, será necesario considerar otras formas para evaluar el grado de elaboración de las explicaciones, como la que utilizan Jacob, Lachner y Scheiter (2021). Estos autores proponen analizar el número de elaboraciones a partir de la identificación de ideas no mencionadas en el material de estudio, que los estudiantes presentan en forma de ejemplos, analogías o experiencias propias.

En el presente artículo, el análisis se ha centrado en el componente verbal de las explicaciones: la narración oral de los videotutoriales y el texto sobreimpreso que los estudiantes hubieran añadido en las imágenes. Sin embargo, la evaluación del grado de elaboración de las explicaciones en productos audiovisuales deberá considerar no solo el componente verbal de la explicación, sino también el componente visual y la relación entre ambos. Como señala Lemke (2002), el lenguaje de las palabras no es suficiente para expresar muchas de las ideas de la ciencia, y es por ello por lo que esta adopta un lenguaje que combina palabras, diagramas, imágenes, gráficas, mapas, ecuaciones, tablas y otras formas de expresión visual y matemática. Tanto el lenguaje escrito como los demás sistemas semióticos, que son culturalmente específicos y aprendidos socialmente, moldean nuestro pensamiento y, por consiguiente, nuestra forma de construir los conceptos científicos (Lemke, 2002; Pérez-Echeverría, Martí y Pozo, 2010; Vygotsky, 1978, 1995).

En cuanto al grado de complejidad de las preguntas de comprensión para la audiencia que los estudiantes añaden a lo largo de sus videotutoriales, los resultados muestran que la gran mayoría de preguntas -casi un $90 \%$ - se sitúan en el nivel inferior de la taxonomía revisada de Bloom: la categoría recordar, es decir, preguntas que solo requieren reconocer o recuperar la respuesta de la memoria. Este porcentaje es parecido al $94 \%$ encontrado por Barbour et al. (2009), pero superior al $61 \%$ identificado por Siko (2013). Estos autores analizaron 1885 y 1250 preguntas, respectivamente, fruto de proyectos en los que estudiantes de secundaria crearon e introdujeron preguntas en juegos hechos con PowerPoint, en ciencias sociales (Barbour et al., 2009) y en química ambiental (Siko, 2013). Curiosamente, solamente en Barbour et al. (2009) -que identificaron un 94\% de preguntas en el nivel inferior- los estudiantes recibieron formación sobre la formulación de preguntas. En cambio, ni en el presente artículo ni en Siko (2013) -que obtuvo un porcentaje menor de preguntas del nivel inferior- los estudiantes no recibieron formación específica sobre cómo generar preguntas complejas. Aun así, cabe señalar que formular este tipo de preguntas -aunque sean del nivel inferior-puede ayudar a los estudiantes a identificar las ideas clave de su explicación. En cualquier caso, los altos porcentajes de preguntas de este nivel sugieren la necesidad de pensar cómo enseñar explícitamente a formular preguntas de niveles superiores, y cómo ofrecer apoyos durante su creación. Los autores también proponen mostrar 
ejemplos variados de preguntas, ofrecer modelos y modelaje, e introducir espacios de evaluación y revisión de las preguntas, también con situaciones de evaluación entre iguales (Barbour et al., 2009; Siko, 2013; Yu y Yang, 2014).

En futuros estudios sobre el proyecto, $u$ otras propuestas similares que consideren la creación de preguntas de comprensión de opción múltiple, se deberá poner el foco no solo en el nivel del enunciado de las preguntas, sino también en la elaboración de las opciones de respuesta -como hacen por ejemplo Tabach y Friedlander (2017) en matemáticas- y en la retroalimentación para la audiencia. En el caso de la retroalimentación, diversos estudios sugieren que hacer que los estudiantes elaboren comentarios para respuestas potenciales de la audiencia tiene beneficios en el uso de estrategias cognitivas y metacognitivas, en la calidad de las preguntas y en las habilidades de toma de perspectiva (Yu y Wu, 2020), así como en la motivación y las emociones académicas positivas (Yu et al., 2018). Zurcher et al. (2016) señalan que elaborar comentarios de retroalimentación ofrece la oportunidad de anticipar concepciones erróneas de la audiencia. Desde la perspectiva de aprenseñar (Duran, 2014), el carácter interactivo de la actividad de elaborar preguntas y retroalimentación para la audiencia podría promover procesos de construcción del conocimiento. De este modo, se complementaría el carácter más expositivo del vídeo.

En resumen, este artículo presenta los primeros resultados del Proyecto Bikos, en el que parejas de estudiantes elaboran videotutoriales cooperativamente a partir de una pregunta sobre el mundo que ellos mismos se plantean. Estos resultados muestran mejoras significativas en el conocimiento específico del contenido, un grado suficiente de elaboración de las explicaciones aproximadamente en dos tercios de la información, y una gran mayoría de preguntas de comprensión orientadas a recordar ideas concretas de la explicación. Hay que poner en valor estos resultados, ya que señalan que los estudiantes fueron capaces de aprender sobre el contenido de sus preguntas al trabajar de forma cooperativa para buscar información y elaborarla -incluso cuando abordaban temas complejos- con el objetivo de crear un videotutorial. La variedad de temas que surgen en el proyecto -y la flexibilidad de la propuesta para ajustarse a cualquier temática- muestra el potencial de la intervención desde la perspectiva de la personalización del aprendizaje (Coll, 2018). En el futuro, sería interesante analizar en profundidad las concepciones erróneas que los estudiantes modifican o mantienen después de haber buscado información y de haber elaborado el videotutorial, para comprender mejor las potencialidades y limitaciones de este tipo de proyectos de indagación documental. Las tareas de indagación documental pueden complementarse muy bien con los proyectos de indagación experimental. De hecho, el planteamiento del Proyecto Bikos permitiría incluir también en el videotutorial la explicación de un experimento, o podría convertirse en el punto de partida para formular hipótesis de cara a un posterior trabajo experimental o de observación. En definitiva, ante la abrumadora cantidad de afirmaciones científicas que recibimos -y buscamos- a diario (Sinatra y Lombardi, 2020), resulta necesario generar situaciones en las que podamos enseñar a los estudiantes a evaluar y a comunicar ideas científicas. La creación de materiales didácticos por parte de los estudiantes -para que otros aprendan, pero también como mecanismo para su propio aprendizaje- puede ofrecer oportunidades de elaborar el conocimiento y de progresar hacia la alfabetización científica. 


\section{Agradecimientos}

Este trabajo ha sido apoyado por el Ministerio de Educación y Formación Profesional a través del contrato FPU18/01663 para la formación de docentes universitarios.

\section{Referencias}

Anderson, L. W. y Bloom, B. S. (2001). A taxonomy for learning, teaching, and assessing: A revision of Bloom's taxonomy of educational objectives. Longman.

Barbour, M., Kromrei, H., McLaren, A., Toker, S., Mani, N. y Wilson, V. (2009). Testing an assumption of the potential of homemade PowerPoint games. En I. Gibson, R. Weber, K. McFerrin, R. Carlsen y D. Willis (Eds.), Proceedings of SITE 2009: Society for Information Technology \& Teacher Education International Conference (pp. 1381-1387). Association for the Advancement of Computing in Education (AACE).

Bargh, J. y Schul, Y. (1980). On the cognitive benefits of teaching. Journal of Educational Psychology, 72(5), 593-604. http://doi.org/10.1037/0022-0663.72.5.593

Bennett, J., Hogarth, S., Lubben, F., Campbell, B. y Robinson, A. (2010). Talking science: The research evidence on the use of small group discussions in science teaching. International Journal of Science Education, 32(1), 69-95. https://doi.org/10.1080/09500690802713507

Benware, C. y Deci, E. (1984). Quality of learning with an active versus passive motivational set. American Educational Research Journal, 21(4), 755-765. http://doi.org/10.3102/00028312021004755

Can, H. B. y Boz, Y. (2016). Structuring cooperative learning for motivation and conceptual change in the concepts of mixtures. International Journal of Science and Mathematics Education, 14(4), 635-657. https://doi.org/10.1007/s10763-014-9602-5

Coiro, J. (2011). Predicting reading comprehension on the Internet: Contributions of offline reading skills, online reading skills, and prior knowledge. Journal of Literacy Research, 43(4), 352-392. https://doi.org/10.1177/1086296X11421979

Coiro, J. y Dobler, E. (2007). Exploring the online reading comprehension strategies used by sixth-grade skilled readers to search for and locate information on the Internet. Reading Research Quarterly, 42(2), 214-257. http://doi.org/10.1598/RRQ.42.2.2

Coll, C. (2013). La educación formal en la nueva ecología del aprendizaje: Tendencias, retos y agenda de investigación. En J. L. Rodríguez (Comp.), Aprendizaje y educación en la sociedad digital (pp. 156-170). Universitat de Barcelona. https://doi.org/10.1344/106.000002060

Coll, C. (2018). Procesos de aprendizaje generadores de sentido y estrategias de personalización. En C. Coll (Coord.), La personalización del aprendizaje (pp. 14-18). Graó.

Coll, C. y Rodríguez, J. L. (2008). Alfabetización, nuevas alfabetizaciones y alfabetización digital: Las TIC en el currículum escolar. En C. Coll y C. Monereo (Eds.), Psicología de la educación virtual (pp. 325-347). Morata.

Colley, C. y Windschitl, M. (2016). Rigor in elementary science students' discourse: The role of responsiveness and supportive conditions for talk. Science Education, 100(6), 1009-1038. https://doi.org/10.1002/sce.21243

Collins, A. y Halverson, R. (2010). The second educational revolution: Rethinking education in the age of technology. Journal of Computer Assisted Learning, 26, 18-27. http://doi.org/10.1111/ j.1365-2729.2009.00339.x

De la Hera, D. P., Sigman, M. y Calero, C. I. (2019). Social interaction and conceptual change pave the way away from children's misconceptions about the Earth. NPJ Science of Learning, 4(1), 1-12. https://doi.org/10.1038/s41539-019-0051-3

Downie, J., Morton, J. A. y McCoustra, M. R. (2017). Bright lights: Big experiments! A public engagement activity for international year of light. Physics Education, 52(1), Article 015005.

Duran, D. (2014). Aprenseñar: Evidencias e implicaciones educativas de aprenderenseñando. Narcea.

Duran, D. (2017). Learning-by-teaching: Evidence and implications as a pedagogical mechanism. Innovations in Education and Teaching International, 54(5), 476-484. http://doi.org/10.10 80/14703297.2016.1156011 
Durán-García, M. E. y Durán-Aponte, E. E. (2013). La termodinámica en los estudiantes de tecnología: Una experiencia de aprendizaje cooperativo. Enseñanza de las Ciencias, 31(1), 45-59. https://doi.org/10.5565/rev/ec/v31n1.614

Eymur, G. y Geban, Ö. (2017). The collaboration of cooperative learning and conceptual change: Enhancing the students' understanding of chemical bonding concepts. International Journal of Science and Mathematics Education, 15(5), 853-871. https://doi.org/10.1007/ s10763-016-9716-z

Farrokhnia, M., Meulenbroeks, R. F. y van Joolingen, W. R. (2020). Student-generated stop-motion animation in science classes: A systematic literature review. Journal of Science Education and Technology, 29, 797-812. https://doi.org/10.1007/s10956-020-09857-1

Fernández-Enguita, M. (2017). Más escuela y menos aula. Morata.

Fiorella, L. y Mayer, R. E. (2014). Role of expectations and explanations in learning by teaching. Contemporary Educational Psychology, 39(2), 75-85. http://doi.org/10.1016/j. cedpsych.2014.01.001

Freire, P. y Faundez, A. (2010). Per una pedagogia de la pregunta. (V. Berenguer y C. Berenguer, Trad.). Edicions del Crec i Denes Editorial. (Obra original publicada en 1985)

Furberg, A. y Arnseth, H. C. (2009). Reconsidering conceptual change from a socio-cultural perspective: Analyzing students' meaning making in genetics in collaborative learning activities. Cultural Studies of Science Education, 4(1), 157-191. https://doi.org/10.1007/s11422-008-9161-6

Gallardo-Williams, M., Morsch, L. A., Paye, C. y Seery, M. K. (2020). Student-generated video in chemistry education. Chemistry Education Research and Practice, 21, 488-495. https:// doi.org/10.1039/C9RP00182D

Decreto 119/2015, de 23 de junio, de ordenación de las enseñanzas de la educación primaria. Diario Oficial de la Generalitat de Catalunya (DOGC), Núm. 6900. Departament d'Ensenyament, Generalitat de Catalunya. https://bit.ly/3AVs9hf

Goldman, S. R., Braasch, J. L., Wiley, J., Graesser, A. C. y Brodowinska, K. (2012). Comprehending and learning from Internet sources: Processing patterns of better and poorer learners. Reading Research Quarterly, 47(4), 356-381. https://doi.org/10.1002/RRQ.027

Hoogerheide, V., Visee, J., Lachner, A. y van Gog, T. (2019). Generating an instructional video as homework activity is both effective and enjoyable. Learning and Instruction, 64, Article 101226. https://doi.org/10.1016/j.learninstruc.2019.101226

Howard, S. K. (2013). Risk-aversion: Understanding teachers' resistance to technology integration. Technology, Pedagogy and Education, 22(3), 357-372. https://doi.org/10.1080/147593 9X.2013.802995

Ilomäki, L., Paavola, S., Lakkala, M. y Kantosalo, A. (2016). Digital competence: An emergent boundary concept for policy and educational research. Education and Information Technologies, 21(3), 655-679. https://doi.org/10.1007/s10639-014-9346-4

Jablonski, D., Hoban, G. F., Ransom, H. S. y Ward, K. S. (2015). Exploring the use of "slowmation" as a pedagogical alternative in science teaching and learning. Pacific-Asian Education, 27(1), 5-20.

Jacob, L., Lachner, A. y Scheiter, K. (2021). Does increasing social presence enhance the effectiveness of writing explanations? PLOS ONE, 16(4), e0250406. https://doi.org/10.1371/journal. pone.0250406

Knight, S. y Mercer, N. (2015). The role of exploratory talk in classroom search engine tasks. Technology, Pedagogy and Education, 24(3), 303-319. https://doi.org/10.1080/1475939X.2014.931884

Kobayashi, K. (2019). Learning by preparing- to- teach and teaching: A meta- analysis. Japanese Psychological Research, 61(3), 192-203. https://doi.org/10.1111/jpr.12221

Lemke, J. L. (2002). Enseñar todos los lenguajes de la ciencia: Palabras, símbolos, imágenes y acciones. En M. Benlloch (Comp.), La educación en ciencias: Ideas para mejorar su práctica. Paidós.

Lin, J. W., Yen, M. H., Liang, J., Chiu, M. H. y Guo, C. J. (2016). Examining the factors that influence students' science learning processes and their learning outcomes: 30 years of conceptual 
change research. Eurasia Journal of Mathematics, Science and Technology Education, 12(9), 2617-2646. https://doi.org/10.12973/eurasia.2016.000600a

Lin, S. W., Liu, Y., Chen, S. F., Wang, J. R. y Kao, H. L. (2016). Elementary school students' science talk ability in inquiry-oriented settings in Taiwan: Test development, verification, and performance benchmarks. International Journal of Science and Mathematics Education, 14(7), 1199-1214. https://doi.org/10.1007/s10763-015-9663-0

Lin, T. J., Lin, T. C., Potvin, P. y Tsai, C. C. (2019). Research trends in science education from 2013 to 2017: Asystematic content analysis of publications in selected journals. International Journal of Science Education, 41(3), 367-387. https://doi.org/10.1080/09500693.2018.1550274

Macedo-Rouet, M., Braasch, J. L., Britt, M. A. y Rouet, J. F. (2013). Teaching fourth and fifth graders to evaluate information sources during text comprehension. Cognition and Instruction, 31(2), 204-226. https://doi.org/10.1080/07370008.2013.769995

Mason, L. y Zaccoletti, S. (2021). Inhibition and conceptual learning in science: A review of studies. Educational Psychology Review, 33, 181-212. https://doi.org/10.1007/s10648-020-09529-x

Mercer, N. (2001). Palabras y mentes: Cómo usamos el lenguaje para pensar juntos. (G. SánchezBarberán, Trad.). Paidós. (Obra original publicada en 2000)

Mills, R., Tomas, L. y Lewthwaite, B. (2019). The impact of student-constructed animation on middle school students'learning about plate tectonics. Journal of Science Education and Technology, 28(2), 165-177. https://doi.org/10.1007/s10956-018-9755-z

Monereo, C. y Fuentes, M. (2008). La enseñanza y el aprendizaje de estrategias de búsqueda y selección de la información en entornos virtuales. En C. Coll y C. Monereo (Eds.), Psicología de la educación virtual (pp. 386-408). Morata.

Muis, K. R., Psaradellis, C., Chevrier, M., Di Leo, I. y Lajoie, S. P. (2016). Learning by preparing to teach: Fostering self-regulatory processes and achievement during complex mathematics problem solving. Journal of Educational Psychology, 108(4), 474-492. http://doi.org/10.1037/ edu0000071

OCDE (2017, Febrero). How does PISA for development measure scientific literacy? PISA for DevelopmentBrief2017/2. https://www.oecd.org/pisa/pisa-for-development/10-How-PISAD-measures-science-literacy.pdf

Ødegaard, M., Arnesen, N. E. y Klette, K. (2016). Talk and use of language in the science classroom: Characteristic features. En K. Klette, O. K. Bergem y A. Roe (Eds.), Teaching and Learning in Lower Secondary Schools in the Era of PISA and TIMSS (pp. 101-112). Springer.

Pavón, F. y Martínez-Aznar, M. M. (2014). La metodología de resolución de problemas como investigación (MRPI): Una propuesta indagativa para desarrollar la competencia científica en estudiantes que cursan un programa de diversificación. Enseñanza de las Ciencias, 32(3), 469-492. http://dx.doi.org/10.5565/rev/ensciencias.1290

Penttilä, J., Kallunki, V., Niemi, H. M. y Multisilta, J. (2016). A structured inquiry into a digital story: Students report the making of a superball. International Journal of Mobile and Blended Learning, 8(3), 19-34. http://doi.org/10.4018/IJMBL.2016070102

Perales, F. J. y Vílchez, J. M. (2015). Iniciación a la investigación educativa con estudiantes de secundaria: El papel de las ilustraciones en los libros de texto de ciencias. Enseñanza de las Ciencias, 33(1), 243-262. http://dx.doi.org/10.5565/rev/ensciencias.1484

Pérez-Echeverría, M., Martí, E. y Pozo, J. I. (2010). Los sistemas externos de representación como herramientas de la mente. Cultura y Educación, 22(2), 133-147. https://doi. org/10.1174/113564010791304519

Petko, D. (2012). Teachers' pedagogical beliefs and their use of digital media in classrooms: Sharpening the focus of the 'will, skill, tool' model and integrating teachers' constructivist orientations. Computers \&Education, 58(4), 1351-1359. https://doi.org/10.1016/j.compedu.2011.12.013

Pozo, J. I. (2014). Psicología del aprendizaje humano: Adquisición de conocimiento y cambio personal. Morata. 
Reigosa, C. y Jiménez, M. P. (2011). Formas de actuar de los estudiantes de laboratorio para la fundamentación de afirmaciones y propuestas de acción. Enseñanza de las Ciencias, 29(1), 23-34. https://doi.org/10.5565/rev/ec/v29n1.128

Reyna, J. y Meier, P. (2018). Learner-generated digital media (LGDM) as an assessment tool in tertiary science education: A review of literature. IAFOR Journal of Education, 6(3), 93-109. https:// doi.org/10.22492/ije.6.3.06

Ribosa, J. y Duran, D. (2021). Student-generated teaching materials: A scoping review mapping the research field [Manuscrito presentado para su publicación]. Departamento de Psicología Básica, Evolutiva y de la Educación, Universitat Autònoma de Barcelona.

Rosa, D. y Martínez-Aznar, M. M. (2019). Resolución de problemas abiertos en ecología para la ESO. Enseñanza de las Ciencias, 37(2), 25-42. https://doi.org/10.5565/rev/ensciencias.2541

Roscoe, R. y Chi, M. (2007). Understanding tutor learning: Knowledge-building and knowledge-telling in peer tutors' explanations and questions. Review of Educational Research, 77(4), 534574. https://doi.org/10.3102/0034654307309920

Siko, J. P. (2013). Are they climbing the pyramid? Rating student-generated questions in a game design project. Canadian Journal of Learning and Technology, 39(1), 1-14. https://doi. org/10.21432/t26k5m

Sinatra, G. M. y Hofer, B. K. (2016). Public understanding of science: Policy and educational implications. Policy Insights from the Behavioral and Brain Sciences, 3(2), 245-253. https://doi. org/10.1177/2372732216656870

Sinatra, G. M. y Lombardi, D. (2020). Evaluating sources of scientific evidence and claims in the posttruth era may require reappraising plausibility judgments. Educational Psychologist, 55(3), 120-131. https://doi.org/10.1080/00461520.2020.1730181

Snelson, C. (2018). Video production in content-area pedagogy: A scoping study of the research literature. Learning, Media and Technology, 43(3), 294-306. http://doi.org/10.1080/174 39884.2018.1504788

Soto, M. (2015). Elementary students' mathematical explanations and attention to audience with screencasts. Journal of Research on Technology in Education, 47(4), 242-258. http://doi. org/10.1080/15391523.2015.1078190

Tabach, M. y Friedlander, A. (2017). Algebraic procedures and creative thinking. ZDM, 49(1), 53-63. https://doi.org/10.1007/s11858-016-0803-y

Topping, K., Buchs, C., Duran, D. y Van Keer, H. (2017). Effective peer learning: From principles to practical implementation. Routledge.

Voogt, J., Fisser, P., Pareja-Roblin, N., Tondeur, J. y van Braak, J. (2013). Technological pedagogical content knowledge: A review of the literature. Journal of Computer Assisted Learning, 29(2), 109-121. https://doi.org/10.1111/j.1365-2729.2012.00487.x

Vygotsky, L. S. (1978). Mind in society: The development of higher psychological processes. (A. R. Luria, M. López-Morillas, M. Cole y J. V. Wertsch, Trad.). Harvard University Press.

Vygotsky, L. S. (1995). Pensamiento y lenguaje. (M. M. Rotger, Trad.). Fausto. (Obra original publicada en 1934)

Webb, M. y Cox, M. (2004). A review of pedagogy related to information and communications technology. Technology, Pedagogy and Education, 13(3), 235-286. https://doi. org/10.1080/14759390400200183

Yeung, K. L., Carpenter, S. K. y Corral, D. (2021). A comprehensive review of educational technology on objective learning outcomes in academic contexts. Educational Psychology Review. Publicación avanzada en línea. https://doi.org/10.1007/s10648-020-09592-4

Yu, F. Y. y Wu, W. S. (2020). Effects of student-generated feedback corresponding to answers to online student-generated questions on learning: What, why, and how? Computers and Education, 145, Article 103723. https://doi.org/10.1016/j.compedu.2019.103723

Yu, F. Y., Wu, W. S. y Huang, H. C. (2018). Promoting middle school students' learning motivation and academic emotions via student-created feedback for online student-created multiple-choice 
questions. Asia-Pacific Education Researcher, 27(5), 395-408. https://doi.org/10.1007/ s40299-018-0398-X

Yu, F. Y. y Yang, Y. T. (2014). To see or not to see: Effects of online access to peer-generated questions on performance. Journal of Educational Technology and Society, 17(3), 27-39.

Zhang, M. y Quintana, C. (2012). Scaffolding strategies for supporting middle school students' online inquiry processes. Computers \& Education, 58(1), 181-196. https://doi.org/10.1016/j. compedu.2014.04.010

Zhu, Z., Bernhard, D. y Gurevych (2009). Amulti-dimensional model for assessing the quality of answers in social QyA sites (Informe núm. TUD-CS-2009-0158). Technische Universität Darmstadt. https://tuprints.ulb.tu-darmstadt.de/1940/1/TR_dimension_model.pdf

Zurcher, D. M., Phadke, S., Coppola, B. P. y McNeil, A. J. (2016). Using student-generated instructional materials in an e-homework platform. Journal of Chemical Education, 93(11), 1871-1878. https://doi.org/10.1021/acs.jchemed.6b00384

\section{Cómo citar en APA:}

Ribosa, J. y Duran, D. (2021). Cuando la curiosidad científica se transforma en un videotutorial para aprender enseñando: conocimiento del contenido, elaboración de las explicaciones y complejidad de las preguntas. Revista Iberoamericana de Educación, 87(2), 85-101. https://doi. org/10.35362/rie8724572 